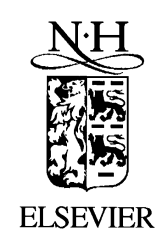

\title{
A driftless gas proportional scintillation counter for muonic hydrogen X-ray spectroscopy under strong magnetic fields
}

\author{
J.F.C.A. Veloso ${ }^{a}$, J.M.F. dos Santos ${ }^{a}$, C.A.N. Conde ${ }^{a} *$, F. Mulhauser ${ }^{b}$, \\ P. Knowles ${ }^{\mathrm{b}}$, C. Donche-Gay ${ }^{\mathrm{b}}$, O. Huot ${ }^{\mathrm{b}}$, D. Taqqu' ${ }^{\mathrm{c}}$, F. Kottmann ${ }^{\mathrm{d}}$ \\ ${ }^{a}$ Physics Department, University of Coimbra, P-3004-516 Coimbra, Portugal \\ ' Institut de Physique de l'Université, CH-1700 Fribourg, Switzerland \\ ${ }^{\mathrm{c}}$ Paul Scherrer Institute, CH-5232 Villigen-PSI, Switzerland \\ ${ }^{\mathrm{d} I n s t i t u t ~ f u ̈ r ~ T e i l c h e n p h y s i k, ~ E T H Z, ~ C H-8093 ~ Z u ̈ r i c h, ~ S w i t z e r l a n d ~}$
}

Received 11 April 2000; received in revised form 20 July 2000; accepted 25 August 2000

\begin{abstract}
An experiment involving muonic hydrogen requires an X-ray detector having large area and working under strong magnetic fields $(5 \mathrm{~T})$ with good energy and timing resolution. A compact, driftless gas proportional scintillation counter (GPSC) capable of operating under such magnetic fields is investigated. This GPSC uses a CsI photocathode deposited onto a microstrip plate as the UV scintillation readout photosensor. This photocathode has the advantage of operating in direct contact with the scintillation gas. The detector is filled with pure xenon and is designed to have a high detection efficiency for $2 \mathrm{keV}$ X-rays. Energy resolutions of $23 \%$ and $22 \%$ were obtained for 1.74 and $2.3 \mathrm{keV}$ X-rays, respectively. The low-energy detector limit due to the electronic noise is $300 \mathrm{eV}$. Its performance in the presence of strong magnetic fields was tested. At magnetic field of $5 \mathrm{~T}$ the detector pulse amplitudes are reduced by less than $25 \%$, while the detector energy resolution and pulse rise time present a relative increase of less than 10\%. (C) 2001 Elsevier Science B.V. All rights reserved.
\end{abstract}

\section{Introduction}

For the standard electronic hydrogen, quantum mechanics predicts that the first excited states $(2 \mathrm{~S}$ and $2 \mathrm{P}$ ) are degenerated. This is not in agreement with experiments since there is a small shift between the $2 \mathrm{~S}$ and the $2 \mathrm{P}$ state, the so-called "Lamb shift", which can only be explained by quantum electrodynamics. The energy levels of muonic hydrogen are 186 times more energetic

\footnotetext{
*Corresponding author. Tel.: + 351-39410654; fax: + 35139829158.

E-mail address: canconde@gian.fis.uc.pt (C.A.N. Conde).
}

than those of the standard (electronic) hydrogen, according to the ratio of reduced masses. The binding energy levels of the muonic hydrogen $1 \mathrm{~S}$ ground state is $2.53 \mathrm{keV}$ and transitions to this state are in the soft X-ray region. If the equivalent Lamb shift can be measured for muonic hydrogen with good accuracy, improved tests of quantum electrodynamics can be obtained and the rms proton radius can be deduced with an accuracy better than 1 part in 1000 .

Such an experiment is being planned in a collaboration involving 8 different institutions [1]. The experiment consists basically in stopping $\mu^{-}$in low-pressure hydrogen under a strong (5 T) 
magnetic field, producing muonic hydrogen. The $2 \mathrm{~S}$ metastable state atoms, then populated, can be excited with a pulsed laser beam to the $2 \mathrm{P}$ state, which decays to the ground state emitting a $1.9 \mathrm{keV}$ X-ray photon. Measuring the number of coincidences between the laser shot and the $1.9 \mathrm{keV}$ photons, as a function of the laser wavelength, the $2 \mathrm{P}-2 \mathrm{~S}$ shift can be obtained.

\section{Rationale}

The X-ray detector for this experiment has to stand strong magnetic fields up to about $5 \mathrm{~T}$, and needs to have a good energy resolution (about $20 \%$ at $2 \mathrm{keV}$ ) with detection areas of about $100 \mathrm{~cm}^{2}$. A standard gas proportional scintillation counter (GPSC) would be the appropriate choice were it not for the photomultiplier tube (PMT) which cannot operate under strong magnetic fields. GPSCs combine room temperature operation with good energy resolution, large detection area, and high counting rates without space charge effects $[2,3]$. Compared to proportional counters or microstrip gas chambers, the superior energy resolution of GPSCs is derived from the very low additional uncertainty introduced by their characteristic amplification process: the production of secondary scintillation rather than charge multiplication [4].

Alternatives to the PMT have been investigated during the past two decades [5-12]. These include multiwire or microstrip proportional chambers with either UV-sensitive filling gas or CsI photocathodes. Recent research work done in Coimbra [9-12] using a microstrip gas chamber (MSGC)based photosensor instead of the photomultiplier tube, will in principle provide a solution for large area, good energy resolution X-ray GPSC working under strong magnetic fields. To provide a small pulse rise time a xenon-driftless-GPSC configuration was chosen. A CsI-covered microstrip plate (MSP), placed within the xenon volume, is used as the GPSC collection grid for the primary electrons and as the amplification stages for the photoelectrons produced due to vacuum ultraviolet (VUV) scintillation. This arrangement must be fairly insensitive to the magnetic fields required for the muonic hydrogen experiment.

In the present work we investigate the performance of a prototype driftless xenon gas proportional scintillation counter based on a MSGC photosensor readout designed to meet the above requirements. Experimental results of tests performed with this detector operating under magnetic fields up to $5 \mathrm{~T}$ are presented.

\section{Experimental system}

The design of this GPSC is schematically illustrated in Fig. 1. The GPSC is a small-scale version of the final detector. Unlike the standard GPSC design with a drift/absorption region, it has only one $0.85-\mathrm{cm}$ deep region in which both absorption and scintillation take place. This is convenient for the measurement of the $1.9 \mathrm{keV}$ $\mathrm{X}$-ray as the absorption depth in xenon at this energy is only $0.8 \mathrm{~mm}$ at 800 Torr or $9.5 \%$ of the depth of the scintillation region and affects the energy resolution only marginally. On the other hand, since the scintillation light is produced immediately after absorption, good time resolution can be achieved. The detector has a closed xenon gas volume. It uses a getter placed in a protect region for gas purification. The $12.5-\mu \mathrm{m}$ thick, $10-\mathrm{mm}$ diameter aluminized Mylar window is maintained at negative voltage, - HV. A Macor ceramic insulates the window holder from the detector body.

The microstrip plate (MSP) is a CERN model MS-4. It consists of $10 \mu \mathrm{m}$ anodes and $80 \mu \mathrm{m}$ cathodes with a $200 \mu \mathrm{m}$ pitch, fabricated of $0.2 \mu \mathrm{m}$ thick chromium film deposited onto a $500 \mu \mathrm{m}$ Desag D263 glass substrate. The backplane is a flat, unstructured layer of $0.1 \mu \mathrm{m}$ chromium. The MSP effective area is $30 \mathrm{~mm} \times$ $30 \mathrm{~mm}$. The backplane and cathodes are maintained at ground potential while a positive voltage of a few hundred volts, $V_{\mathrm{a}}$, is applied to the anodes.

The absorption produces an electron cloud near the GPSC entrance window. As the electron drift toward the microstrip they excite the xenon atoms and induce ultraviolet $(170 \mathrm{~nm})$ luminescence. The 


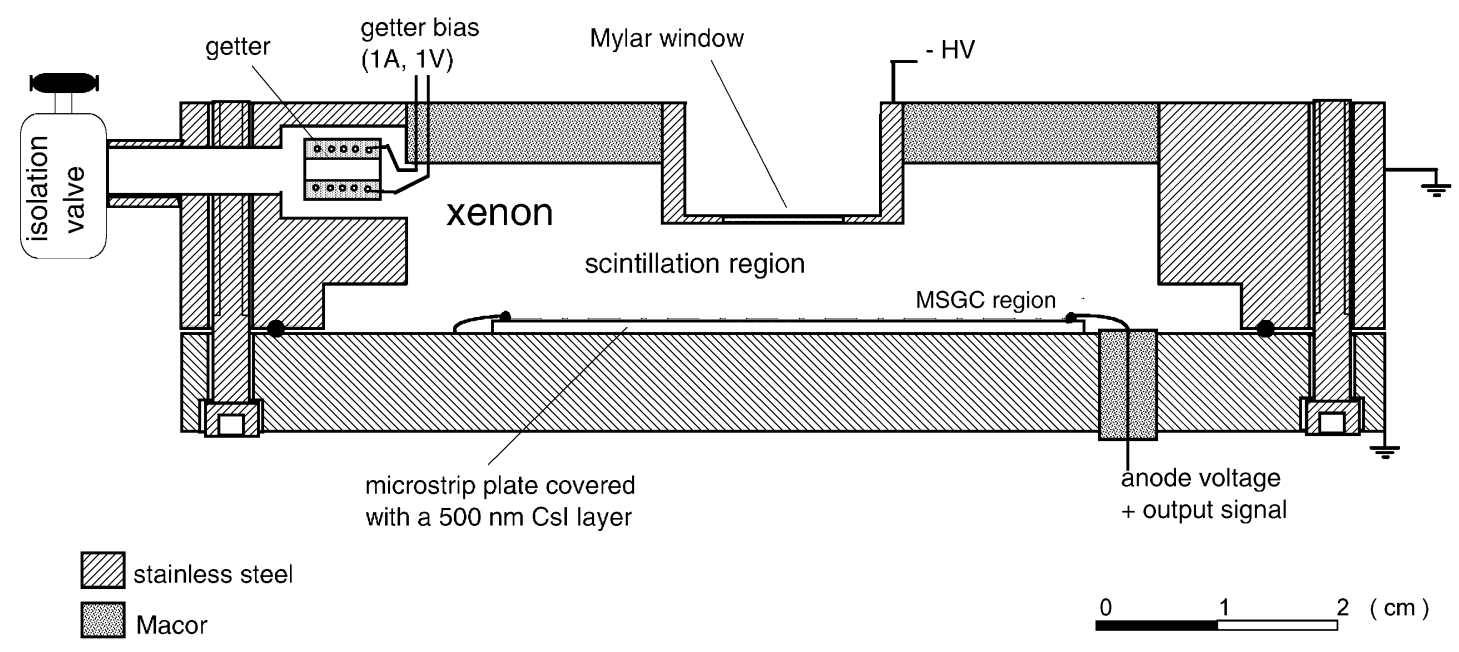

Fig. 1. Schematic of the driftless GPSC with a CsI microstrip plate photosensor.

loss of primary electrons in X-ray interaction near the entrance window is minimized by the high electric field present in the scintillation region [13].

A $500 \mathrm{~nm}$ layer of high-purity CsI film is vacuum evaporated onto the MSP surface. The xenon scintillation photons eject photoelectrons from the CsI deposited on the cathode strips. These electrons drift towards the anode strips where multiplication occurs due to the strong electric field present in this region, leading to a fairly large pulse proportional to the energy absorbed in the GPSC main volume.

\section{The influence of the magnetic field}

Fig. 2 shows the detector orientation relative to the electric $\boldsymbol{E}$ and magnetic $\boldsymbol{B}$ field. For an electron cloud drifting in the scintillation region, the effect of a magnetic field on the electron energy distribution is described by a factor in the differential equation for the electron energy distribution function, which is given by [14]

$\left[1+\frac{e^{2} B^{2} \lambda^{2}}{2 m_{\mathrm{e}} \varepsilon}\right]$

where $e$ and $m_{\mathrm{e}}$ are the electron charge and mass, respectively, $\varepsilon$ is the electron energy, $B$ the magnetic field and $\lambda$ the electron mean free path

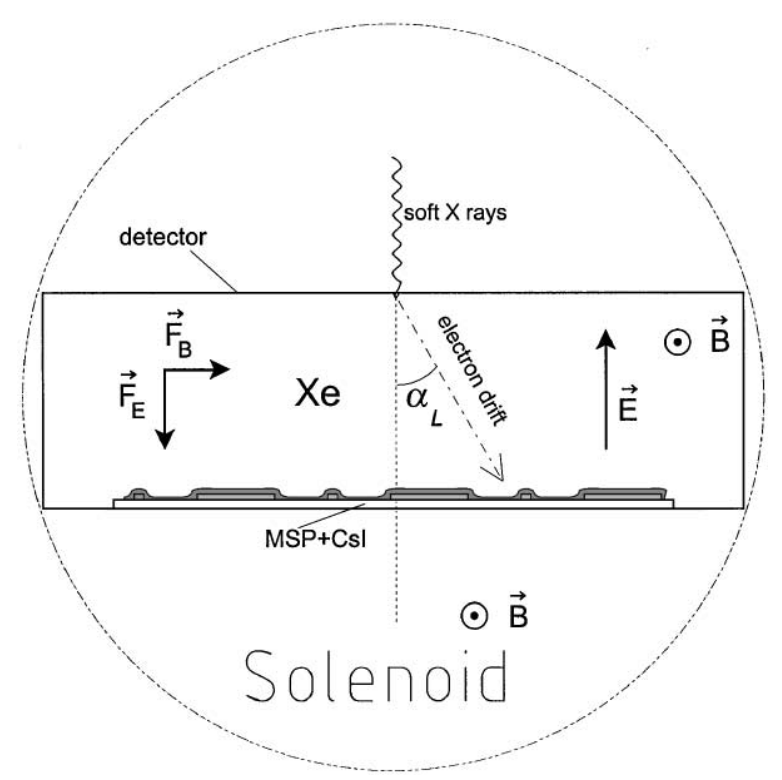

Fig. 2. Schematic of the detector orientation relatively to the electric $\boldsymbol{E}$ and magnetic $\boldsymbol{B}$ fields.

between collisions. For a typical reduced electric field (electric field intensity divided by the gas pressure, $E / p$ ) of $5 \mathrm{~V} \mathrm{~cm}^{-1} \mathrm{Torr}^{-1}$ in the GPSC scintillation region, ${ }^{1} \varepsilon$ is about $3.6 \mathrm{eV}$ and $\lambda$ about

\footnotetext{
${ }^{1} 1$ Torr $=133$ pa.
} 
$1.6 \times 10^{-7} \mathrm{~m}[15]$. Thus, for $B=5 \mathrm{~T}$

$\frac{e^{2} B^{2} \lambda^{2}}{2 m_{\mathrm{e}} \varepsilon}=1.55 \times 10^{-2} \ll 1$

which means that, under these conditions, the magnetic field does not affect significantly the electron energy distribution function and thus the GPSC secondary scintillation yield.

On the other hand, for the same field intensities the electron drift velocity, $v_{\mathrm{d}}$, is about $1 \mathrm{~cm} \mu \mathrm{s}^{-1}$ [15] and the Lorentz angle (Fig. 2)

$\alpha_{\mathrm{L}}=\operatorname{arctg}\left(\frac{\left|\boldsymbol{v}_{\mathrm{d}} \times \boldsymbol{B}\right|}{|\boldsymbol{E}|}\right)$

will be about $5^{\circ}$, a value too small to have a significant effect on the detector pulse duration and on the solid angle effects in light collection. Also, it has been found that the MSGC charge multiplication process is little dependent on strong magnetic fields [16].

Another factor that can be sensitive to magnetic fields is the CsI photocathode quantum efficiency (QE): a photoelectron can return to the photocathode surface due to the Lorentz force. The photoelectron leaving the photocathode with a velocity, $v$, perpendicular to the magnetic field and to the surface, under an electric field $\boldsymbol{E}$ orthogonal to this surface, will describe a cycloid trajectory described by the parametric equation, as a function of time, $t$,

$x=\frac{b}{\omega} \sin (\omega t-\theta)+\frac{E_{y}}{B}+\frac{b}{\omega} \sin (\theta)$

$y=\frac{b}{\omega} \cos (\omega t-\theta)+\frac{b}{\omega} \cos (\theta)$

where $y$ is the coordinate parallel to the electric field $\boldsymbol{E}$, and $x$ the coordinate parallel to surface and perpendicular to $\boldsymbol{E}$ and $\boldsymbol{B}$. The other constants are given by $\omega=e B / m_{\mathrm{e}}$,

$b=-\frac{E_{y}}{B \cos (\theta)}$, and $\theta=\arctan \left(-\frac{v B}{E_{y}}\right)$.

If the electron mean free path is much shorter than the cycloid length, the probability of photoelectrons being scattered by the gas atoms and escaping from the photocathode surface is high, and the magnetic field should have negligible influence on the photocathode QE. Otherwise, the QE should decrease with increasing magnetic field because some photoelectrons will return to the photocathode surface.

The length of the trajectory, $s$, from the starting point, to the point where the electrons meets again the surface $(y=0)$

$S=\int_{t=0}^{t(y=0)} \sqrt{\dot{x}^{2}+\dot{y}^{2}} \mathrm{~d} t$

was calculated by numerical integration and is given for various values of $B$ in Table 1, assuming that the photoelectron leaves the photocathode with an average energy of $0.4 \mathrm{eV}[17,18]$, an $E_{y} / p$ of $10.4 \mathrm{~V} \mathrm{~cm}^{-1}$ Torr $^{-1}$ above the photocathode surface [12], and a xenon pressure of 1100 Torr. As the photoelectron mean-free path is only $\sim 0.58 \mu \mathrm{m}$ for the above conditions [19], rather smaller than the calculated trajectory length (Table 1), photoelectrons have a good probability of being scattered by the xenon atoms before returning again to the surface. Thus, the CsI film QE will not be much affected even for 5 T magnetic fields. Piuz et al. [20] have found a reduction of $10 \%$ in the quantum efficiency for a reflective CsI photocathode operating in a $\mathrm{CH}_{4}$ environment under a magnetic field of $0.9 \mathrm{~T}$. However, the operation conditions were different, namely the electric field intensity at the photocathode surface.

\section{Detector performance characteristics}

The MSP signals are fed through a low-noise $1.5 \mathrm{~V} / \mathrm{pC}$ charge-integrated pre-amplifier and a spectroscopy amplifier, with shaping times of $1 \mu \mathrm{s}$, to a 1024 multichannel analyzer. For pulse amplitude and energy resolution measurements, the X-ray pulse-height distributions are fitted to a

\section{Table 1}

Cycloid trajectory length $s$, of a $0.4 \mathrm{eV}$ photoelectron, perpendicularly emitted from CsI photocathode, as a function of magnetic field, considering an orthogonal electric field of $10.4 \mathrm{~V} \mathrm{~cm}^{-1}$ Torr $^{-1}$ above the photocathode surface, from the starting point, to the point where the electron meets again the surface, assuming it does not collide with the gas molecules

\begin{tabular}{llllll}
\hline$B(\mathrm{~T})$ & 1 & 2 & 3 & 4 & 5 \\
$S\left(10^{-6} \mathrm{~m}\right)$ & 52.8 & 13.7 & 6.5 & 3.9 & 2.7 \\
\hline
\end{tabular}


Gaussian function superimposed on a linear background, from which the centroid and the full-width at half-maximum are determined.

Fig. 3 depicts the detector pulse amplitude and energy resolution as a function of the reduced electric field in the scintillation region for 1.74 and $5.9 \mathrm{keV} \mathrm{X}$-rays and for an anode voltage of $350 \mathrm{~V}$. The results exhibit the characteristic behavior of the xenon-filled GPSC secondary scintillation yield with a threshold at about $1 \mathrm{~V} \mathrm{~cm}^{-1} \mathrm{Torr}^{-1}$ and an exponential increase for $E / p$ above $8 \mathrm{~V} \mathrm{~cm}^{-1}$ Torr $^{-1}$, reflecting the presence of charge multiplication in the scintillation region.

For standard GPSCs the electron luminescence yield increases linearly with $E / p$ [4]. In this detector, the deviation from the linear trend characteristic of GPSC electroluminescence yield is attributed to the decrease of the electric field intensity at the CsI surface, on the cathode strips, as the $E / p$ increases in the scintillation region. This effect results in a slight reduction of the photoelectron extraction efficiency [12]. The energy resolution improves with $E / p$ up to $9 \mathrm{Vcm}^{-1} \mathrm{Torr}^{-1}$. Above this value a degradation of the energy resolution is noticeable due to additional fluctuations introduced by charge multiplication.

The energy resolution at $5.9 \mathrm{keV}$ comes out to be only slightly better than at $1.74 \mathrm{keV}$ because of the

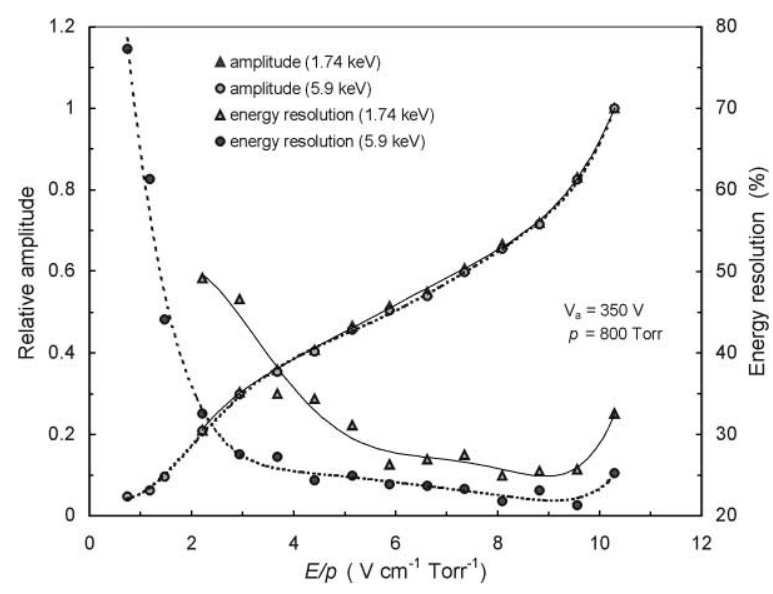

Fig. 3. Detector pulse amplitude and energy resolution as a function of the reduced electric field $E / p$ in the scintillation region for 1.74 and $5.9 \mathrm{keV} \mathrm{X-rays} \mathrm{and} \mathrm{for} \mathrm{an} \mathrm{anode} \mathrm{voltage} \mathrm{of}$ $350 \mathrm{~V}$. much longer absorption depth at $5.9 \mathrm{keV}$ in xenon. It amounts to $2.6 \mathrm{~mm}$ (at 800 Torr) and results in an low-energy tail in the pulse-height distribution having an exponential shape of $21 \%$ half-width. For a gas pressure of 1100 Torr the detector energy resolution improve to $17 \%$ for $5.9 \mathrm{keV} \mathrm{X-rays}$ while for $1.74 \mathrm{keV}$ improvement remains small, reflecting the already small penetration depth of these X-rays.

In Fig. 4 we present the detector pulse amplitude and energy resolution as a function of the microstrip anode voltage for $5.9 \mathrm{keV} \mathrm{X}$-rays and for a reduced electric field of $8 \mathrm{~V} \mathrm{~cm}^{-1}$ Torr $^{-1}$ in the scintillation region. The exponential increase of the detector amplitude as a function of the anode voltage, typical of MSGCs, is observed [21]. However, amplitude deviation from the exponential behavior due to positive UV photon feedback occurs for $V_{\mathrm{a}}$ voltages above $330 \mathrm{~V}$. The energy resolution is rather worse than the $12 \%$ figure obtained before [12] due to the different absorption position of the $\mathrm{X}$-ray in this driftless GPSC.

In Fig. 5 we depict the X-ray pulse-height distributions obtained from pure $\mathrm{Si}$ and $\mathrm{S}$ targets excited with a ${ }^{55} \mathrm{Fe} \mathrm{X}$-ray source, for full detector window irradiation. The targets were $30 \mathrm{~cm}$ diameter discs positioned at $45^{\circ}$ to the detector axis and $1 \mathrm{~cm}$ away from the detector window. The pulse-height distributions depicted in Fig. 5 were obtained for different amplifier gains. The spectral

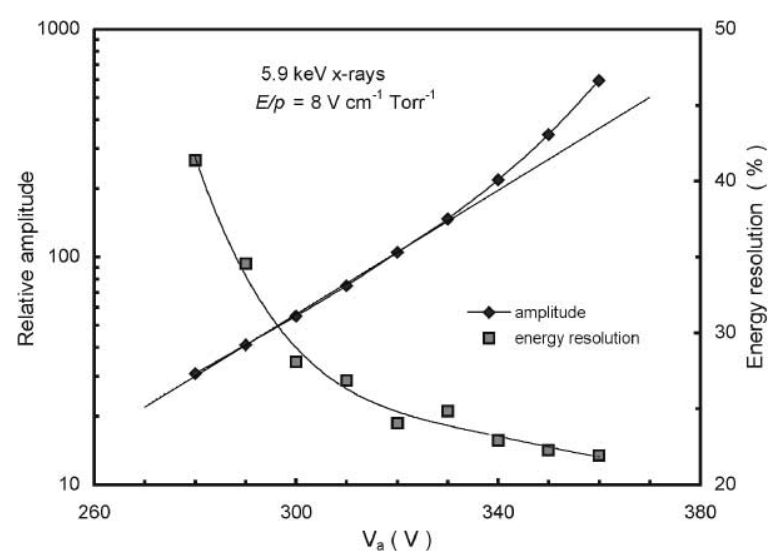

Fig. 4. Detector pulse amplitude and energy resolution as a function of the microstrip anode voltage for $5.9 \mathrm{keV}$ X-rays and for a reduced electric field of $8 \mathrm{~V} \mathrm{~cm}^{-1} \mathrm{Torr}^{-1}$. 

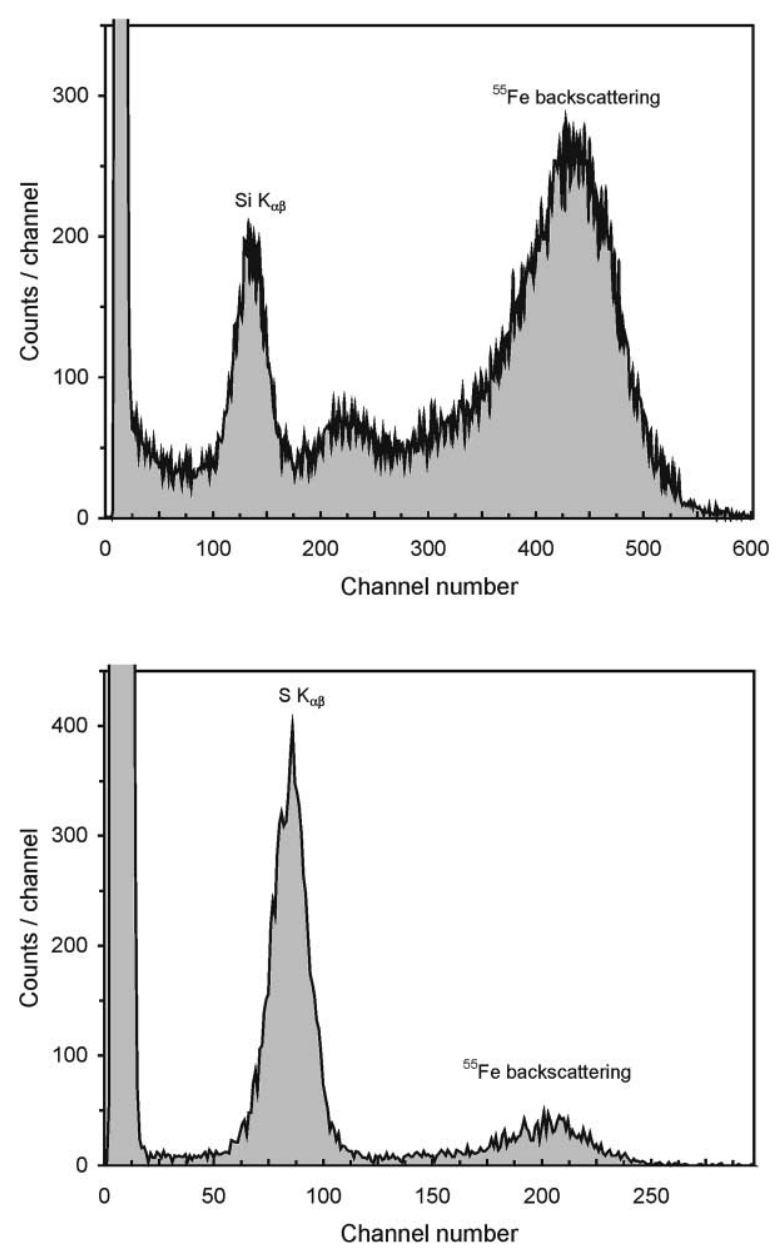

Fig. 5. Pulse-height distributions obtained from pure silicon and sulfur targets irradiated with a ${ }^{55} \mathrm{Fe} \mathrm{X}$-ray source, using different amplifier gains for the two targets.

features include the element $\mathrm{K}$ line, the source backscattered X-rays and the electronic noise tail. Energy resolutions of $23 \%$ and $22 \%$ were obtained for 1.74 and $2.30 \mathrm{keV} \mathrm{X}$-rays, respectively. These values compare favorably with the value of $\sim 30 \%$ obtained with a xenon-filled MSGC for $1.74 \mathrm{keV}$ $\mathrm{X}$-rays [21]. The low-energy detection limit due to electronic noise is $300 \mathrm{eV}$.

\section{Detector operation under strong magnetic fields}

The experimental tests for the detector operation under strong magnetic fields were carried out at the Paul Scherrer Institute (PSI), Switzerland. The detector was placed inside a cryogenic superconducting solenoid, capable of achieving magnetic field up to $5 \mathrm{~T}$. This solenoid is $1 \mathrm{~m}$ long with a bore hole at room temperature of $20 \mathrm{~cm}$ diameter. The magnetic field is homogeneous $\left(10^{-4}\right)$ over an axial distance of $30 \mathrm{~cm}$ from the center. For these tests the detector was filled at 1100 Torr.

The detector orientation inside the solenoid was chosen in such a way that the MSP electrodes were placed parallel to the magnetic field direction, Fig. 2. This is the most unfavorable position for the CsI-MSP operation since the photoelectron drift direction towards the strip anodes is perpendicular to the magnetic field.

For the measurements an X-ray fluorescence source was used. It was made of sulfur excited with a ${ }^{55} \mathrm{Fe} X$-ray source. Due to a restriction related with the available high-voltage supplies, a maximum reduced electric field of $5.5 \mathrm{~V} \mathrm{~cm}^{-1} \mathrm{Torr}^{-1}$ was used in the scintillation region during the experiments. However, this restraint does not invalidate a correct assessment of the detector behavior in the presence of the magnetic field.

The detector pulse amplitude was monitored as a function of the magnetic field for the 2.3 and $5.9 \mathrm{keV} \mathrm{X}$-rays and for reduced electric field intensities of 3.3 and $5.5 \mathrm{~V} \mathrm{~cm}^{-1} \mathrm{Torr}^{-1}$ in the scintillation region, while the MSP $V_{\text {a }}$ voltage was kept at $370 \mathrm{~V}$. The same variation was found for both X-ray energies and both reduced electric field intensities.

In Fig. 6 we depict the relative average amplitude of the detector pulses as a function of the magnetic field for the above conditions. The effect of the magnetic field results in a reduction of the detector pulse amplitude, achieving a maximum variation of $25 \%$ for $5 \mathrm{~T}$. This reduction can be attributed to solid angle effects, due to the Lorentz angle together with the small size of the photocathode and to a reduction in the photocathode quantum efficiency, as referred to in Section 2. However, this variation is less than the one obtained in Ref. [20] $(10 \%$ at $0.9 \mathrm{~T})$, probably because the reduced electric field at the CsI surface in the cathode strips, about $10 \mathrm{~V} \mathrm{~cm}^{-1} \mathrm{Torr}^{-1}$, is more favorable for the photoelectron extraction 


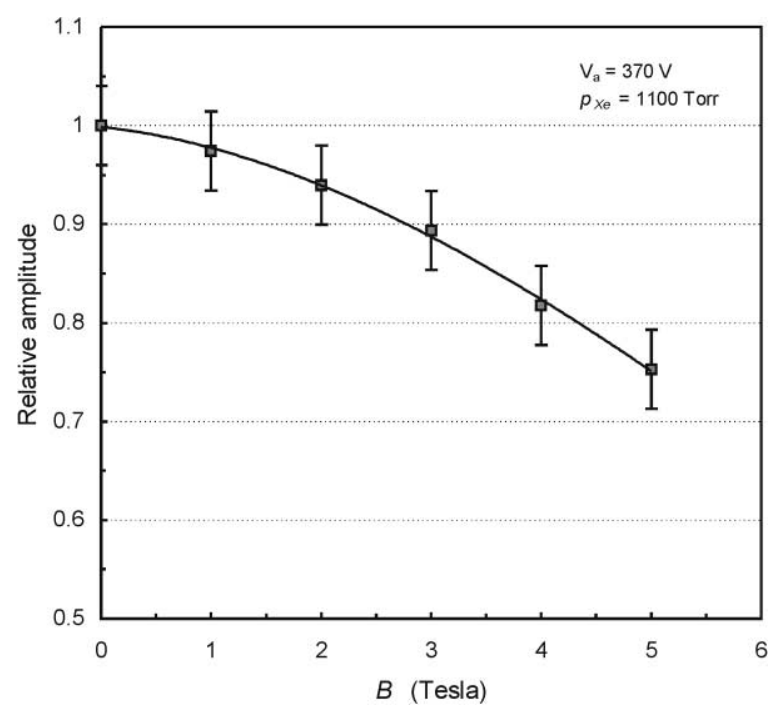

Fig. 6. Average of the relative detector pulse amplitude as function of magnetic field for anode voltage of $370 \mathrm{~V}$.

under magnetic fields than the reduced electric field of $4 \mathrm{~V} \mathrm{~cm}^{-1} \mathrm{Torr}^{-1}$ used in that reference.

The detector energy resolution for the 2.3 and $5.9 \mathrm{keV} \mathrm{X}$-rays as a function of the magnetic field is presented in Fig. 7 for $E / p=5.5 \mathrm{~V} \mathrm{~cm}^{-1} \mathrm{Torr}^{-1}$. The energy resolution increases slightly with the magnetic field up to a maximum variation of about $10 \%$ at $5 \mathrm{~T}$. This correlates reasonably well with the observed decrease in signal amplitude.

In Fig. 8 the measured pulse-height distributions are presented for detector operation under 0 and under $5 \mathrm{~T}$ magnetic field and for a $E / p=5.5 \mathrm{~V} \mathrm{~cm}^{-1} \mathrm{Torr}^{-1}$. As shown, the amplitude of the detector pulses is reduced by about $25 \%$. Fig. 8 also shows that the detector electronic noise and peak-to-background ratios are almost independent of the magnetic field.

The detector pulses rise time was monitored as a function of the magnetic field for the $2.3 \mathrm{keV}$ $\mathrm{X}$-rays and for an $E / p=5.5 \mathrm{~V} \mathrm{~cm}^{-1} \mathrm{Torr}^{-1}$. A digital oscilloscope was used to read the pulses directly from the pre-amplifier signal output, using a discrimination window for the amplitudes corresponding to the $2.3 \mathrm{keV} \mathrm{X}$-rays. An average of 400 pulses was considered for each rise-time measurement. This allows to average out of the random electronic noise superimposed on each

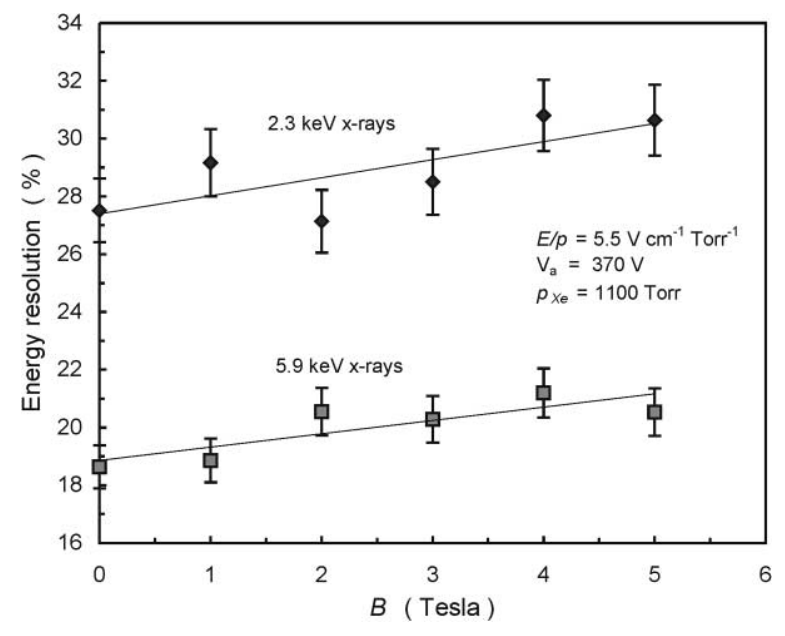

Fig. 7. Energy resolution of the detector for 2.3 and $5.9 \mathrm{keV}$ $X$-rays as a function of the magnetic field for $E / p=5.5 \mathrm{~V} \mathrm{~cm}^{-1} \mathrm{Torr}^{-1}$ and a anode voltage of $370 \mathrm{~V}$.

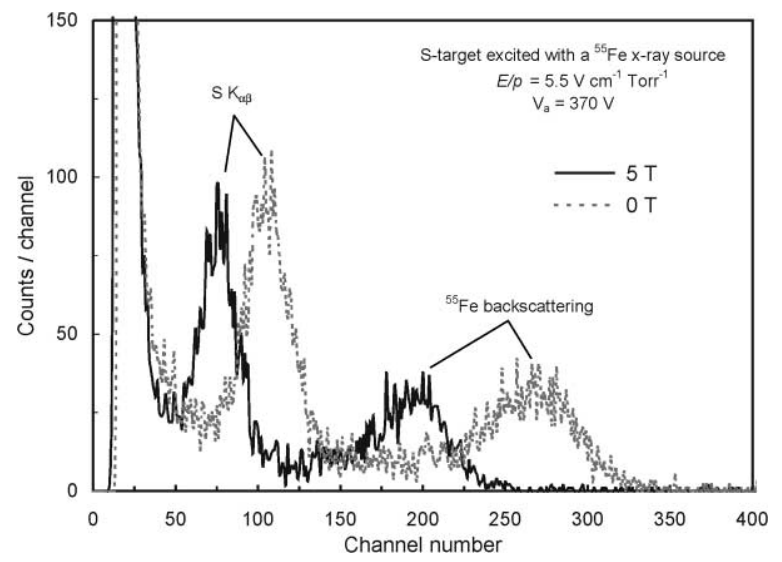

Fig. 8. Pulse-height distributions obtained from the sulfur target excited with a ${ }^{55} \mathrm{Fe} \mathrm{X}$-ray source for detector operation under 0 and $5 \mathrm{~T}$ magnetic field and for an $E / p=5.5 \mathrm{~V} \mathrm{~cm}^{-1} \mathrm{Torr}^{-1}$.

individual pulse. Fig. 9 depicts a typical average pulse obtained with the digital oscilloscope, for a magnetic field of $5 \mathrm{~T}$. The vertical dashes marked on the average pulse represent $10 \%$ and $90 \%$ of the maximum amplitude value and the pulse rise time considered was the time interval between these points. This rise time corresponds to the primary electron drift time while crossing the scintillation region. 


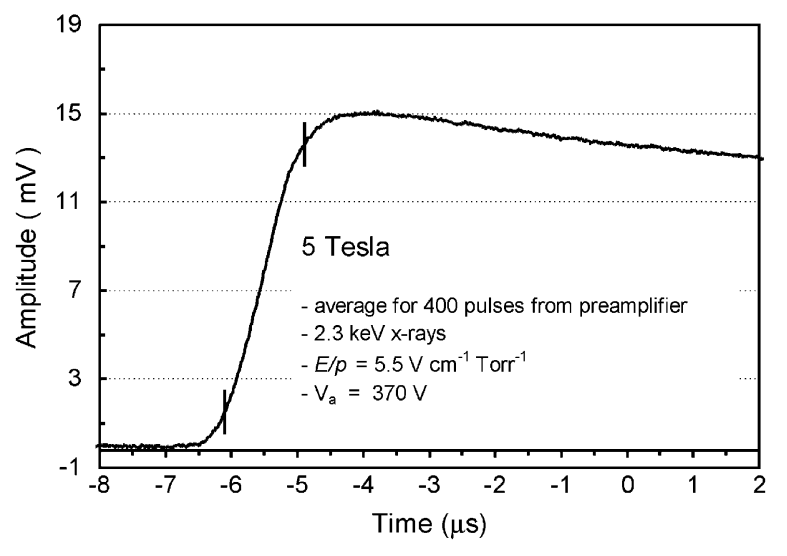

Fig. 9. Average of 400 pulses of $2.3 \mathrm{keV}$ read directly from the preamplifier with a digital oscilloscope for a $5 \mathrm{~T}$ magnetic field, an $E / p=5.5 \mathrm{~V} \mathrm{~cm}^{-1} \mathrm{Torr}^{-1}$ and an anode voltage of $370 \mathrm{~V}$.

Fig. 10 depicts the rise time of the average pulse as a function of the magnetic field for $2.3 \mathrm{keV}$ $\mathrm{X}$-rays. For comparison, Fig. 10 also shows the calculated values of the primary electron cloud drift time, $t_{\mathrm{d}}$, according to the equation

$$
t_{\mathrm{d}}(B)=\frac{t_{\mathrm{d}}(0)}{\cos \left[\alpha_{\mathrm{L}}(B)\right]}
$$

assuming that the electron drift velocity is independent of the magnetic field and $t_{\mathrm{d}}(0)$ the measured drift time of the average pulse for $0 \mathrm{~T}$ magnetic field, taking also into account the longer drift trajectory of the electron cloud, due to the Lorentz angle (Fig. 2), derived from Eq. (2). The calculated results lead to a negligible rise-time dependence on the magnetic field due to the small Lorentz angle of the drifting electrons in the xenon scintillation region. This trend agrees with the experimental results up to magnetic fields of $4 \mathrm{~T}$. The experimental result obtained for $5 \mathrm{~T}$ magnetic field departs about $10 \%$ from that behavior and it is not yet fully understood. Further studies to investigate the detector behavior at this magnetic field intensity are planned.

The detector time resolution has not yet been investigated in much detail. The available data indicates that it is mainly limited by the electronic noise. In the present configuration a preliminary analysis of the measured rise-time fluctuations

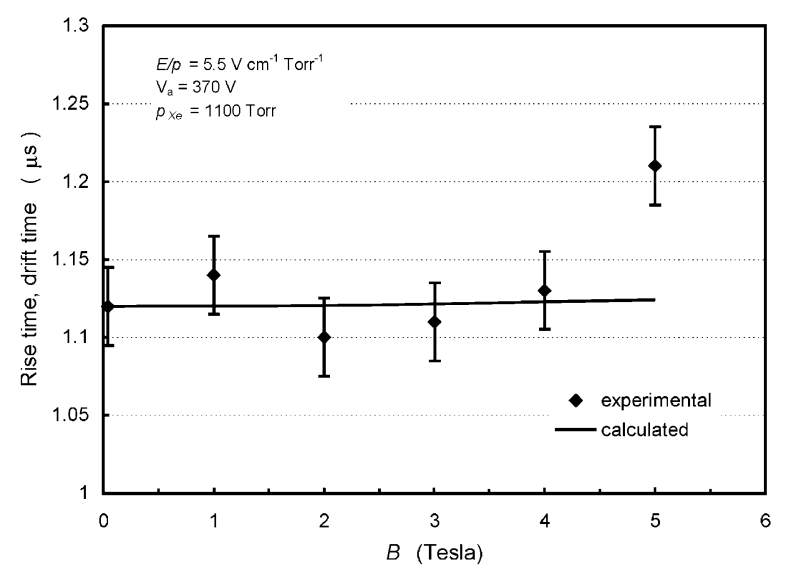

Fig. 10. Rise time of the average pulses (Fig. 9) and calculated values for the primary electron cloud drift time as a function of magnetic field.

indicates that less than $200 \mathrm{~ns}$ time resolution is achievable at $2 \mathrm{keV}$.

\section{Conclusions}

A driftless gas proportional scintillation counter for soft X-rays using a CsI-covered microstrip plate as a VUV photosensor presents good characteristics for operating under strong magnetic fields. At magnetic fields of $5 \mathrm{~T}$ the detector pulse amplitudes are reduced by less than $25 \%$, while the detector energy resolution and pulse rise time present a relative increase of less than $10 \%$.

Energy resolutions of $23 \%$ and $22 \%$ were obtained for 1.74 and $2.3 \mathrm{keV}$ X-rays, respectively. The low-energy detection limit due to electronic noise is $300 \mathrm{eV}$. The energy resolutions achieved with this prototype for low-energy X-rays are better than the ones obtained with a proportional counter, but still well above the ones obtained with a standard GPSC instrumented with PMTs.

Improvements can be achieved by increasing the cathode width of the MSP (presently $80 \mu \mathrm{m}$ ) relative to the pitch (presently $200 \mu \mathrm{m}$ ). Alternatively a new design where the driftless GPSC is coupled to a P10-filled MSGC is under investigation. It should reduce the positive feedback and allow a significantly greater MSGC gain improving both energy and time resolution. 
The performances of the present detector configuration are well adapted to the muonic hydrogen spectroscopy experiment. A version with large detection area is presently being implemented for the observation of the muonic hydrogen K-X-ray energy and time distributions.

This detector has the required performance for the muonic hydrogen X-ray spectroscopy that motivated its development.

\section{Acknowledgements}

The detector development work was carried out in the Atomic and Nuclear Instrumentation Group of the Instrumentation Center (Unit 217/94) of the Physics Department, University of Coimbra. The studies of the performance of this detector under strong magnetic field was carried out at the Paul Scherrer Institute, in Switzerland. We thank to J. Pinheiro da Providência, Paulo Rachinhas and Luis Távora for helpful discussions. Supported is acknowledge to Fundação para a Ciência e a Tecnologia (FCT) through Project PRAXIS/P/ FIS/13140/1998, and to Swiss National Science Foundation.

\section{References}

[1] D. Taqqu et al., Hyperfine Interactions 119 (1999) 311.

[2] A. Smith, M. Bavdaz, Rev. Sci. Instr. 63 (1992) 683.
[3] I.V. Varvaritsa, V.V. Vikulov, M.A. Ivashov, V.I. Panov, K.I. Filatov, K.I. Schekin, Instr. Exp. Tech. 35 (1992) 745.

[4] F.I.G.M. Borges, J.M.F. dos Santos, T.H.V.T. Dias, F.P. Santos, P.B.J.M. Rachinhas, C.A.N. Conde, Nucl. Instr. and Meth. A 422 (1999) 321.

[5] A.J.P.L. Policarpo, Nucl. Instr. and Meth. 153 (1978) 389.

[6] D.F. Anderson, Nucl. Instr. and Meth. 178 (1980) 125.

[7] D.G. Simons, P.A.J. de Korte, A. Peacock, A. Smith, J.A.M. Bleeker, IEEE Trans. Nucl. Sci. NS-32 (1985) 345.

[8] V. Dangendorf, A. Breskin, R. Chechik, H. Schmidt-Bocking, Nucl. Instr. and Meth. A 289 (1990) 322.

[9] J.F.C.A. Veloso, J.A.M. Lopes, J.M.F. dos Santos, C.A.N. Conde, IEEE Trans. Nucl. Sci. NS-43 (1996) 1232.

[10] J.A.M. Lopes, J.M.F. dos Santos, C.A.N. Conde, R.E. Morgado, IEEE Trans. Nucl. Sci. NS-44 (1997) 517.

[11] J.F.C.A. Veloso, J.M.F. dos Santos, C.A.N. Conde, Nucl. Instr. and Meth. A 422 (1999) 273.

[12] J.F.C.A. Veloso, J.M.F. dos Santos, C.A.N. Conde, Nucl. Instr. and Meth. A 457 (2001) 253.

[13] T.H.V.T. Dias, F.P. Santos, A.D. Stauffer, C.A.N. Conde, Phys. Rev. A 46 (1992) 237.

[14] M. Fátima, A. Ferreira, C.A.N. Conde, N. Ayres de Campos, J.M.S.C. Gil, IEEE Trans. Nucl. Sci. NS-27 (1980) 208.

[15] T.H.V.T. Dias, F.P. Santos, A.D. Stauffer, C.A.N. Conde, Phys. Rev. A 48 (1993) 2887.

[16] F. Angelini et al., Nucl. Instr. and Meth. A 343 (1994) 441.

[17] H. Di Stefano, W.E. Spicer, Phys. Rev. B 7 (1973) 1554.

[18] A. Akkermann, T. Boutboul, A. Breskin, R. Chechik, Gibrekhterman, J. Appl. Phys. 76 (1994) 4656.

[19] P.J.B.M. Rachinhas, J.A.M. Lopes, T.H.V.T. Dias, F.P. Santos, C.A.N. Conde and A.D. Stauffer, Photoelectron collection efficiency in a xenon atmosphere: a Monte Carlo study, Presented at 1999-IEEE Nuclear Science Symposium, 24-30 October, Seattle, USA.

[20] F. Piuz et al., Nucl. Instr. and Meth. A 433 (1999) 178.

[21] J.F.C.A. Veloso, J.M.F. dos Santos, C.A.N. Conde, R.E. Morgado, X-Ray Spectrom. 26 (1997) 237. 